\title{
Electronic clinical decision support system (eCDSS) in the management of asthma: from theory to practice
}

\author{
Jean Bousquet $\mathbb{1}^{1,2,3,4,5,6}$ \\ Affiliations: ${ }^{1}$ MACVIA-France, Fondation partenariale FMC VIA-LR, Montpellier, France. ${ }^{2}$ INSERM U 1168 , \\ VIMA, Ageing and chronic diseases epidemiological and public health approaches, Villejuif, France. \\ ${ }^{3}$ Université Versailles St-Quentin-en-Yvelines, UMR-S 1168, Montigny le Bretonneux, France. "Euforea, \\ Brussels, Belgium. ${ }^{5}$ Charité, Universitätsmedizin Berlin, Humboldt-Universität zu Berlin, Berlin, Germany. \\ ${ }^{6}$ Berlin Institute of Health, Comprehensive Allergy Center, Dept of Dermatology and Allergy, Berlin, Germany.
}

Correspondence: Jean Bousquet, CHU Montpellier, 371 Avenue du Doyen Gaston Giraud, 34295 Montpellier Cedex 5, France. E-mail: jean.bousquetdorange.fr

@ERSpublications

Electronic decision support systems may improve asthma control http://ow.ly/UoAe30o6IEC

Cite this article as: Bousquet J. Electronic clinical decision support system (eCDSS) in the management of asthma: from theory to practice. Eur Respir J 2019; 53: 1900339 [https://doi.org/10.1183/13993003.003392019].

Controlling symptoms while minimising the side-effects of treatment is the main aim of asthma management. If a patient's asthma stabilises, the clinician should then consider stepping down the treatment [1]. In practice, the implementation of step-down or step-up strategies includes 1) the use of risk prediction, 2) tools to support shared decision-making and 3) communication between clinicians and patients about risk. Major contributors to poor asthma control include the following evidence-based care gaps [2]: 1) monitoring of control, resulting in the under-recognition of suboptimal control, 2) adjustment of medication, and 3) delivery of an asthma action plan. Asthma action plans, combined with asthma education and regular follow-up, can improve the symptoms and quality of life of the patient and can reduce hospitalisation [3]. However, it is difficult and time-consuming to regularly assess control in primary care. mHealth has the potential to transform the face of healthcare and one of its benefits can be to provide a clinical decision support system (CDSS).

A CDSS is a health information technology system designed to assist clinicians and other health care professionals in clinical decision-making. In medicine, CDSSs have become a major topic in artificial intelligence. According to the National Academy of Medicine (Washington, DC, USA) [4], "facilitative clinical decision support is a practical necessity for every clinician in our rapidly-evolving health and healthcare landscape." A CDSS can reduce the burden that exponentially expanding clinical knowledge and care complexity places on clinicians, other healthcare professionals or patients.

CDSSs provide clinicians and other health care professionals with knowledge and person-specific information, intelligently filtered or presented at appropriate times, to enhance health and healthcare. CDSSs can enhance decision-making through the use of the following tools: 1) computerised alerts and reminders to healthcare providers and patients, 2) clinical guidelines, 3) focused patient data reports and 4) diagnostic support [5].

Several self-management mHealth tools exist for asthma. Interventions such as the mobile app, the smart inhaler, the handheld asthma monitoring device, and SMSs were used to improve asthma control $[6,7]$. 
However, the electronic clinical decision support system (eCDSS) is less well approached. A systematic review on asthma, published in 2014, concluded that the current generation of CDSSs was unlikely to result in improving outcomes for asthma patients due to the fact that they are rarely used and that advice is not followed [8]. Studies showed inconsistent outcomes and little insight into the functionalities required for mHealth asthma interventions. This underlined the need for systematic intervention design. However, none of these interventions corresponded to a real CDSS. Future CDSSs need to align better with professional workflows in order to enable easily accessible, pertinent and timely advice within the consultation.

CDSSs are based on health information technology that should deliver the right information, at the right point and format within the decision and care processes. Outcomes would then be optimised by consistently applying the best available knowledge for every patient's needs and goals [4]. mHealth tools such as apps or tablets may be useful in supporting CDSSs for the management of asthma $[9,10]$.

An eCDSS now exists in allergic rhinitis [11]. It is based on MASK (Mobile Airways Sentinel NetworK) $[12,13]$. Certain studies have used CDSSs in a variety of settings for adults and children with chronic airway disease $[14,15]$. However, to our current knowledge, there has previously been no validated eCDSS for the management of asthma that showed a clinically relevant impact [8]. The study reported by GUPTA et al. [16] in the current issue of the European Respiratory Journal has filled this gap, and the paper reports the clinical impact of a series of mHealth tools from the same group. Major gaps in evidence-based asthma practice exist in primary care. Targeted knowledge translation interventions are required in order to address these gaps. They can be tailored by leveraging the identified behavioural predictors [17]. The same group designed a touch tablet asthma questionnaire while identifying patient preferences for usability features of such questionnaires [18]. An evidence-based prototype was created and a rapid-cycle design (semi-structured focus group testing, analysis, corresponding modifications, re-testing) was employed on asthma patients. The group developed a computerised CDSS (the Electronic Asthma Management System; eAMS) to address the major care gaps and sought to measure its impact on care in adults with asthma. Overall, the CDSS development included medication de-escalation rules according to asthma guidelines and the establishment of evidence-based rules for the control of asthma through systematic review.

The study reported by GUPTA et al. [16] was a 2-year interrupted time series study of usual care (year 1) versus eAMS (year 2). It took place in three Canadian primary care sites and included asthmatic adults who had received asthma medication over the past 12 months. The eAMS consisted of a touch tablet patient questionnaire that was completed in the waiting room. Its real-time data processing resulted in an electronic medical record-integrated clinician decision support. The primary outcome was the delivery of an action plan that increased the number of eligible patients from 0 to $18 \%$. Physician visits with action plan delivery increased by $30.5 \%$. The level of assessment of asthma control increased from $5 \%$ to $28 \%$. Clinicians escalated controller therapy in $3.2 \%$ of baseline visits versus $126 / 3240$ (3.9\%) of intervention visits $(\mathrm{p}=0.12)$. At baseline, a short-acting beta-agonist alone was added in 62 visits and a controller was added in 54 visits. With the intervention, this occurred in 33 and 229 visits $(\mathrm{p}<0.001)$.

This study is extremely significant as it demonstrates the applicability of eAMS to primary care. However, it also has the following defects.

The severity of asthma could not be appreciated and further studies are required to clarify this point. It is possible that the magnitude of the effect will increase if patients with severe asthma are studied. However, the tool is designed for primary care and many patients in that setting have milder disease. The primary outcome was the delivery of the asthma action plan. However, it is unknown as to whether this resulted in more significant clinical outcomes.

More generally, in order to be fully effective, the CDSS must integrate with the clinical workflow of a healthcare organisation. It would appear that the eAMS is a stand-alone tool that lacks interoperability with reporting and EHR software. Another potential problem related to the CDSS is alert fatigue for clinicians. Neither clinician nor patient satisfaction were assessed.

The eAMS improved the quality of care in asthma in real-world primary care settings. Strategies to further increase clinician uptake, randomised controlled trials and real world evidence are required in order to assess the impact on health outcomes and effectiveness. However, this study paves the way for the implementation of an eCDSS to improve asthma control.

Conflict of interest: J. Bousquet reports personal fees for advisory board work, consultancy and honoraria for lectures from Chiesi, Cipla, Hikma, Menarini, Mundipharma, Mylan, Novartis, Sanofi-Aventis, Takeda, Teva and Uriach, and has shares in Kyomed, outside the submitted work. 


\section{References}

1 Gionfriddo MR, Hagan JB, Rank MA. Why and how to step down chronic asthma drugs. BMJ $2017 ; 359$ : j4438.

2 To T, Guttmann A, Lougheed MD, et al. Evidence-based performance indicators of primary care for asthma: a modified RAND Appropriateness Method. Int J Qual Health Care 2010; 22: 476-485.

3 Kouri A, Kaplan A, Boulet LP, et al. New evidence-based tool to guide the creation of asthma action plans for adults. Can Fam Physician 2019; 65: 103-106.

4 Tcheng J, Bakken S, Bates DW, et al. Optimizing Strategies for Clinical Decision Support: Summary of a Meeting Series. The Learning Health System Series. Washington, National Academy of Medicine, 2017. Available from: www.healthit.gov/topic/safety/clinical-decision-support

5 Clinical Decision Support. HealthITgov. 2018. www.healthit.gov/topic/safety/clinical-decision-support Date last updated: April 10, 2018.

6 Hui CY, Walton R, McKinstry B, et al. The use of mobile applications to support self-management for people with asthma: a systematic review of controlled studies to identify features associated with clinical effectiveness and adherence. J Am Med Inform Assoc 2017; 24: 619-632.

7 Simpson AJ, Honkoop PJ, Kennington E, et al. Perspectives of patients and healthcare professionals on mHealth for asthma self-management. Eur Respir J 2017; 49: 1601966.

8 Matui P, Wyatt JC, Pinnock H, et al. Computer decision support systems for asthma: a systematic review. NPJ Prim Care Respir Med 2014; 24: 14005.

9 Rudin RS, Fanta CH, Predmore Z, et al. Core components for a clinically integrated mHealth app for asthma symptom monitoring. Appl Clin Inform 2017; 8: 1031-1043.

10 Tinschert P, Jakob R, Barata F, et al. The potential of mobile apps for improving asthma self-management: a review of publicly available and well-adopted asthma apps. JMIR Mhealth Uhealth 2017; 5: e113.

11 Courbis AL, Murray RB, Arnavielhe S, et al. Electronic clinical decision support system for allergic rhinitis management: MASK e-CDSS. Clin Exp Allergy 2018; 48: 1640-1653.

12 Bousquet J, Anto JM, Annesi-Maesano I, et al. POLLAR: Impact of air POLLution on Asthma and Rhinitis a European Institute of Innovation and Technology Health (EIT Health) project. Clin Transl Allergy 2018 ; 8: 36.

13 Bousquet J, Arnavielhe S, Bedbrook A, et al. MASK 2017: ARIA digitally-enabled, integrated, person-centred care for rhinitis and asthma multimorbidity using real-world-evidence. Clin Transl Allergy 2018; 8: 45.

14 Cornick R, Picken S, Wattrus C, et al. The Practical Approach to Care Kit (PACK) guide: developing a clinical decision support tool to simplify, standardise and strengthen primary healthcare delivery. BMJ Glob Health 2018; 3: Suppl. 5, e000962.

15 Picken S, Hannington J, Fairall L, et al. PACK Child: the development of a practical guide to extend the scope of integrated primary care for children and young adolescents. BMJ Glob Health 2018; 3: Suppl. 5, e000957.

16 Gupta S, Price C, Agarwal G, et al. The Electronic Asthma Management System (eAMS) improves primary care asthma management. Eur Respir J 2019; 53: 1802241.

17 Price C, Agarwal G, Chan D, et al. Large care gaps in primary care management of asthma: a longitudinal practice audit. BMJ Open 2019; 9: e022506.

18 Lam Shin Cheung V, Kastner M, Sale JE, et al. Development process and patient usability preferences for a touch screen tablet-based questionnaire. Health Informatics J 2019; 1460458218824749. 